\title{
When are Borel functions Baire functions?
}

\author{
by
}

\author{
Mogens Fosgerau ( Lyngby)
}

\begin{abstract}
The following two theorems give the flavour of what will be proved.
THEOREM. Let $Y$ be a complete metric space. Then the families of first Baire class functions and of first Borel class functions from [0,1] to $Y$ coincide if and only if $Y$ is connected and locally connected.

THEOREM. Let $Y$ be a separable metric space. Then the families of second Baire class functions and of second Borel class functions from [0,1] to $Y$ coincide if and only if for all finite sequences $U_{1}, \ldots, U_{q}$ of nonempty open subsets of $Y$ there exists a continuous function $\phi:[0,1] \rightarrow Y$ such that $\phi^{-1}\left(U_{i}\right) \neq \emptyset$ for all $i \leq q$.
\end{abstract}

0. Introduction. Given metric spaces $X$ and $Y$ we let $\mathcal{B} a_{0}(X, Y)$ be the family of all continuous functions from $X$ to $Y$. For all ordinals $0<\alpha<\omega_{1}$ we define the Baire class $\alpha$, denoted by $\mathcal{B} a_{\alpha}(X, Y)$, to be the family of all limits of pointwise convergent sequences of functions from $\bigcup_{\beta<\alpha} \mathcal{B} a_{\beta}(X, Y)$. A class $\alpha$ Borel function from $X$ to $Y\left(0<\alpha<\omega_{1}\right)$ is a function $f$ such that $f^{-1}(G)$ is a Borel set of additive class $\alpha$ whenever $G$ is an open subset of $Y$. For reference on Borel sets see [10]. We denote the family of all class $\alpha$ Borel functions by $\mathcal{B} o_{\alpha}(X, Y)$.

The first Baire and Borel classes do not coincide in general. The function $f:[0,1] \rightarrow\{0,1\}$ defined by $f(1)=1$ and $f(t)=0$ when $t<1$ is of first Borel class, but clearly is not of first Baire class.

The Lebesgue-Hausdorff Theorem in [10, p. 391] tells us that if $X$ is metric and if $Y$ is an $n$-dimensional cube, $[0,1]^{n}, n \in \mathbb{N}$, or the Hilbert cube, $[0,1]^{\mathbb{N}}$, then the first Baire and Borel classes of functions from $X$ to $Y$ do coincide.

More general theorems of this kind has been proved. Rolewicz showed in [14] that if $Y$ is a separable convex subset of a normal linear space, then the first Baire and Borel classes of functions from $X$ to $Y$ coincide. In [6] Hansell gave an extension of the Lebesgue-Hausdorff Theorem asserting that, if every continuous function from a closed subset of $X$ to $Y$ can be 
extended continuously to $X$, then every $\sigma$-discrete (see Section 2 ) first Borel class function from $X$ to $Y$ is also of first Baire class. It was pointed out that Hansell's proof was incomplete, but that it was valid whenever the range space is an absolute extensor for metric spaces, hence when $Y$ is any convex subset of a locally convex linear topological space by the BorsukDugundji Extension Theorem. A proof of this is also given in [9] where the domain space is only assumed to be collectionwise normal (Hausdorff). If, in addition, the range is separable, then the domain need only be normal (see [9, Theorem 1.2 and the subsequent Remark 1.3]). Hansell remarks that this latter result appears to be new but it was known to M. Laczkowich earlier [12]. In [13] Rogers gave another corrected version of Hansell's statement, namely that if every continuous function from a closed subset of $X$ to $Y$ can be extended continuously to $X$, and if, for each point $y$ in $Y$ and each neighbourhood $L$ of $y$, there is a second neighbourhood $N$ of $y$ such that, for each closed subset $F$ of $X$ and each continuous map $f$ from $F$ to $N$, there is a continuous extension of $f$ mapping $X$ into $L$, then every $\sigma$-discrete first Borel class function from $X$ to $Y$ is also of first Baire class.

In the present paper we prove that all $\sigma$-discrete first Borel class functions from a metric space into a metric, arcwise connected and locally arcwise connected space are of first Baire class. We then look for a converse to this result and prove the following theorem. We write $I=[0,1]$ and $\Sigma(X, Y)$ for the class of $\sigma$-discrete functions from $X$ to $Y$.

TheOREM 2. Let $Y$ be complete metric. Then the following three statements are equivalent: (i) $Y$ is connected and locally connected; (ii) $\mathcal{B} a_{1}(I, Y)$ $=\mathcal{B} o_{1}(I, Y)$; and (iii) $\mathcal{B} a_{1}(X, Y)=\mathcal{B} o_{1}(X, Y) \cap \Sigma(X, Y)$ for all metric spaces $X$.

Having considered the case for the first Baire and Borel classes, we then turn our attention to the higher classes. Here the classic theorem is the Banach Theorem which can be found in [10] or in [1]. This theorem uses the concept of an analytically representable function. The analytically representable functions of class one are the first Borel class functions, and the analytically representable functions of class $\alpha$ are the functions which are pointwise limits of analytically representable functions of classes lower than $\alpha$. The Banach Theorem tells us that if $X$ is metric and if $Y$ is separable and metric then the set of analytically representable functions of class $\alpha$ coincides with the set of Borel class $\alpha(\alpha+1)$ functions when $\alpha$ is finite (infinite). Another theorem by Banach in [1] states that if $Y$ is also arcwise connected then the set of Baire class $\alpha$ functions can replace the analytically representable functions of class $\alpha$ in the Banach Theorem for $\alpha \geq 2$. In [2] Brown showed in particular that if $X$ is compact metric and $Y$ is arcwise connected, separable and metric, then the class of all Borel functions 
and the class of all Baire functions coincide. In [6] Hansell generalized the Banach Theorem to the case where $Y$ need not be separable, using the notion of a $\sigma$-discrete function. Hansell's result states that if $Y$ is metric and $f: X \rightarrow Y$ is $\sigma$-discrete and of Borel class $\alpha(\alpha+1)$ then $f$ is analytically representable of class $\alpha$, whenever $\alpha$ is finite (infinite).

Again we find that a very simple "connectedness" condition tells us when the Baire and Borel classes coincide. We shall prove the following theorem.

THEOREM 3. Let $Y$ be a separable metric space. The following statements are equivalent: (i) For all finite sequences $U_{1}, \ldots, U_{q}$ of nonempty open subsets of $Y$, there exists a continuous function $\phi: I \rightarrow Y$ with $\phi^{-1}\left(U_{i}\right) \neq \emptyset$ for all $i \leq q$; (ii) $\mathcal{B} a_{2}(X, Y)=\mathcal{B}_{o_{2}}(X, Y)$ for all metric spaces $X$; (iii) For each metric space $X$ and for each finite (countable, infinite) ordinal $\alpha \geq 2$, $\mathcal{B} a_{\alpha}(X, Y)$ coincides with $\mathcal{B} o_{\alpha}(X, Y)\left(\mathcal{B} o_{\alpha+1}(X, Y)\right)$.

In [2] Brown makes a remark which implies that if $Y$ is a separable metric space the condition (i) implies that $\bigcup_{\alpha>0} \mathcal{B} a_{\alpha}(X, Y)=\bigcup_{\alpha>0} \mathcal{B} o_{\alpha}(X, Y)$ for all metric spaces $X$. His remark seems to imply the converse. He gives proof of neither of these results.

This paper was written at University College London. The author wishes to express his thanks to his supervisors David Preiss, C. A. Rogers and John Jayne. The helpful comments from the referee are also thankfully acknowledged.

1. Definitions. A space is said to be locally arcwise connected if each point of the space has an arbitrarily small (not necessarily open) arcwise connected neighbourhood. This is equivalent to requiring that, for all $\varepsilon$, every point of the space must have an open neighbourhood such that any two points of the neighbourhood can be joined with an arc of diameter less than $\varepsilon$.

We say that a family of sets $\mathcal{A}$ refines a second family of sets $\mathcal{B}$ if each set of $\mathcal{A}$ is contained in a set of $\mathcal{B}$, and $\cup \mathcal{A}=\bigcup \mathcal{B}$. We write this $\mathcal{A} \prec \mathcal{B}$. Given any set $A$ in a metric space $X$ and $\varepsilon>0$, we denote the generalized open ball with "centre" $A$ and radius $\varepsilon$ as $B(A, \varepsilon)=\{x \in X: d(x, A)<\varepsilon\}$.

2. $\sigma$-discrete functions. We shall make use of the notion of a $\sigma$ discrete function, as developed by A. H. Stone and R. W. Hansell, to allow us to consider general metric spaces, using techniques normally used for separable metric spaces. In this section the necessary definitions and results concerning $\sigma$-discrete functions will be given. The reader is referred to $[5]-[7]$ for further information. All spaces are assumed to be metric.

A family of sets in a topological space is said to be discrete if each point of the space has a neighbourhood that meets at most one set of the 
family. The family is said to be uniformly discrete if there exists $\varepsilon>0$ such that the distance between any two sets of the family is greater than $\varepsilon$. A family of sets is said to be $\sigma$-discrete if it can be decomposed into countably many subfamilies, each of which is discrete. A family of sets is said to be $\sigma$-uniformly discrete if it can be decomposed into countably many uniformly discrete families. By [5, Lemma 2 and its proof], if $\mathcal{A}$ is a $\sigma$-discrete family of $\mathcal{F}_{\sigma}$-sets, then there exists a $\sigma$-uniformly discrete family $\mathcal{B}$ of $\mathcal{F}_{\sigma}$-sets such that $\mathcal{B} \prec \mathcal{A}$.

A family of sets is a base for a function from one topological space into another if the pre-image of any open set is a union of sets from the family. A function is said to be $\sigma$-discrete if it has a $\sigma$-discrete base. The family of all $\sigma$-discrete functions from $X$ to $Y$ is denoted by $\Sigma(X, Y)$. In any metric space there exists a $\sigma$-discrete family of open sets, forming a base for the topology (see [10, p. 235]). Using this it can be shown that any continuous map with metric range is $\sigma$-discrete. The family $\Sigma(X, Y)$ is closed under pointwise limits ([7]), so all Baire class $\alpha$ functions, $\alpha<\omega_{1}$, are $\sigma$-discrete. In [10, p. 386], it is shown that functions of Baire class $\alpha$ are of Borel class $\alpha$, respectively $\alpha+1$, according as $\alpha$ is finite or infinite. In [6] it is shown that a $\sigma$-discrete Borel class $\alpha$ function, where $\alpha \geq 2$, is the pointwise limit of a sequence of $\sigma$-discrete Borel functions, each of class strictly lower than $\alpha$. Hence, if for some $\alpha \geq 1, \mathcal{B} a_{\alpha}(X, Y)$ is equal to $\mathcal{B} o_{\alpha}(X, Y) \cap$ $\Sigma(X, Y)$, respectively $\mathcal{B} o_{\alpha+1}(X, Y) \cap \Sigma(X, Y)$, according as $\alpha$ is finite or infinite, then we deduce that $\mathcal{B} a_{\beta}(X, Y)$ is equal to $\mathcal{B} o_{\beta}(X, Y) \cap \Sigma(X, Y)$ $\left(\mathcal{B} o_{\beta+1}(X, Y) \cap \Sigma(X, Y)\right)$ for all finite (infinite) ordinals $\beta$ greater than $\alpha$. A $\sigma$-discrete function of the first Borel class from one metric space into another has a $\sigma$-discrete closed base $([6])$. Every function from a metric space to a separable metric space is $\sigma$-discrete, and every Borel function from a space that is a Souslin- $\mathcal{F}$ set in some complete metric space to a metric space is $\sigma$-discrete $([6])$. It is consistent with and independent of ZFC to assume that all Borel functions from a metric space to a metric space are $\sigma$-discrete (see [4]). Under Martin's Axiom plus the negation of the Continuum Hypothesis there exists an uncountable $X \subset \mathbb{R}$ each subset of which is a relative $\mathcal{F}_{\sigma}$-set. Thus any one-to-one map from $X$ onto a discrete subset of a suitably large hedgehog space (see [3, p. 314]) is an example of a Borel class 1 map which is not Baire class 1 .

3. First Baire class functions. We shall start with two lemmas. The first is purely technical.

LEMMA 1. Let $\mathcal{A}$ be a $\sigma$-discrete family of closed sets covering the metric space $X$. Then there exist families $\mathcal{B}^{p}, p=1,2, \ldots$, of closed sets such that:

- $\bigcup_{p} \mathcal{B}^{p} \prec \mathcal{A}$; 
- each member of $\mathcal{B}^{p}$ is contained in some member of $\mathcal{B}^{p+1}$, for all $p$;

- $\mathcal{B}^{p}$ is uniformly discrete for all $p$; and

- $\bigcup_{p} \mathcal{B}^{p}=X$.

Pr o of. Write $\mathcal{A}$ as the countable union of discrete families $\mathcal{A}_{k}$ of closed sets, and, for each $j$, let $\mathcal{C}_{j}=\left\{A \backslash \bigcup_{k<j} \cup \mathcal{A}_{k}: A \in \mathcal{A}_{j}\right\}$ and $\mathcal{C}=\bigcup_{j} \mathcal{C}_{j}$. Then $\mathcal{C}$ is a disjoint, $\sigma$-discrete family of $\mathcal{F}_{\sigma}$-sets such that $\mathcal{C} \prec \mathcal{A}$. By $[5$, Lemma 2 and its proof], we can write each $C \in \mathcal{C}$ as an increasing union of $\mathcal{F}_{\sigma}$-sets, $C=\bigcup_{n} D_{C}^{n}$, where the families $\left\{D_{C}^{n}: C \in \mathcal{C}\right\}$ are uniformly discrete for all $n$. Write each $D_{C}^{n}=\bigcup_{m} F_{C}^{n m}$, where the sets $F_{C}^{n m}$ are closed. Then the families $\mathcal{B}^{p}=\left\{\bigcup_{n, m \leq p} F_{C}^{n m}: C \in \mathcal{C}\right\}, p \in \mathbb{N}$, satisfy the conclusion of the lemma.

Our second lemma provides us with approximating functions to a given function.

Lemma 2. Let $X$ be a metric space and let $Y$ be a metric and arcwise connected space. Let $f: X \rightarrow Y$ be given and let $\mathcal{D}_{n}, n=1, \ldots, p$, be families of nonempty closed sets in $X$ such that:

- $\mathcal{D}_{n}$ is uniformly discrete for all $n$;

- each member of $\mathcal{D}_{n+1}$ is contained in some member of $\mathcal{D}_{n}$ for all $n<p ;$ and

- $x_{1}, x_{2} \in A \in \mathcal{D}_{n}$ implies that $f\left(x_{1}\right)$ and $f\left(x_{2}\right)$ can be joined with an arc of diameter less than $2^{-n}$.

Then there exists a continuous function $g: X \rightarrow Y$ such that if $x \in \bigcup \mathcal{D}_{n}$, $n \leq p$, then $d(f(x), g(x)) \leq 2^{-n+2}$.

P r o of. Since the families $\mathcal{D}_{n}, n \leq p$, are uniformly discrete, we can find $\varepsilon_{1}>2 \varepsilon_{2}>\ldots>2^{p-1} \varepsilon_{p}>0$ so that $\left\{B\left(A, \varepsilon_{n}\right): A \in \mathcal{D}_{n}\right\}$ is a discrete family of open sets for each $n \leq p$. Observe that, for $A \subset C$ with $A \in \mathcal{D}_{n+1}$ and $C \in \mathcal{D}_{n}$, we have $B\left(A, \varepsilon_{n+1}\right) \subset B\left(C, \varepsilon_{n} / 2\right)$. Pick $y^{\prime} \in f(X)$ and $y_{A} \in f(A)$ for all $A \in \bigcup_{n \leq p} \mathcal{D}_{n}$. For each $A \in \mathcal{D}_{1}$ let $\phi_{A}^{1}: I \rightarrow Y$ be an arc with $\phi_{A}^{1}(0)=y^{\prime}$ and $\phi_{A}^{1}(1)=y_{A}$. For each $A \in \mathcal{D}_{n}, 1<n \leq p$, there is a unique $C \in \mathcal{D}_{n-1}$ with $A \subset C$, since the family $\mathcal{D}_{n-1}$ is disjoint. Let $\phi_{A}^{n}: I \rightarrow Y$ be an arc of diameter at most $2^{-n+1}$, with $\phi_{A}^{n}(0)=y_{C}$ and $\phi_{A}^{n}(1)=y_{A}$.

We shall define a sequence $g_{0}, g_{1}, \ldots, g_{p}$ of continuous functions from $X$ to $Y$ and arrange that the function $g=g_{p}$ satisfies the requirements of the lemma. We start the inductive process by taking $g_{0}(x)=y^{\prime}$ for all $x$ in $X$. Write

$$
D_{1}=\bigcup\left\{\overline{B\left(A, \varepsilon_{1} / 2\right)}: A \in \mathcal{D}_{1}\right\}, \quad E_{1}=X \backslash \bigcup\left\{B\left(A, \varepsilon_{1}\right): A \in \mathcal{D}_{1}\right\} .
$$

Since the family $\left\{B\left(A, \varepsilon_{1}\right): A \in \mathcal{D}_{1}\right\}$ is discrete, $D_{1}$ and $E_{1}$ are disjoint 
closed sets. Write

$$
h_{1}(x)=\frac{d\left(x, E_{1}\right)}{d\left(x, D_{1}\right)+d\left(x, E_{1}\right)},
$$

so that $h_{1}$ is a continuous function on $X$ taking value 1 on $D_{1}$ and 0 on $E_{1}$. Set

$$
g_{1}(x)= \begin{cases}g_{0}(x)=y^{\prime} & \text { on } E_{1}, \\ y_{A} & \text { on } \overline{B\left(A, \varepsilon_{1} / 2\right)} \text { for each } A \in \mathcal{D}_{1}, \\ \phi_{A}^{1} \circ h_{1}(x) & \text { on } B\left(A, \varepsilon_{1}\right) \backslash \overline{B\left(A, \varepsilon_{1} / 2\right)} \text { for each } A \in \mathcal{D}_{1} .\end{cases}
$$

Since the sets $E_{1}, \overline{B\left(A, \varepsilon_{1} / 2\right)}, B\left(A, \varepsilon_{1}\right) \backslash \overline{B\left(A, \varepsilon_{1} / 2\right)}$, for $A \in \mathcal{D}_{1}$, are disjoint with union $X$, the function $g_{1}$ is well-defined. We verify that $g_{1}$ is continuous on $X$. For $x_{0} \in X$ we can choose a neighbourhood $N$ of $x_{0}$ that meets at most one set of the family $\left\{\overline{B\left(A, \varepsilon_{1}\right)}: A \in \mathcal{D}_{1}\right\}$. If $N$ meets none of these sets then $g_{1}(x)=g_{0}(x)$ for $x$ in $N$, and $g_{1}$ is continuous at $x_{0}$. Suppose that $N$ meets $\overline{B\left(A, \varepsilon_{1}\right)}$ for some $A$ in $\mathcal{D}_{1}$, but meets no $\overline{B\left(A^{\prime}, \varepsilon_{1}\right)}$ with $A^{\prime} \in \mathcal{D}_{1}$, $A^{\prime} \neq A$. Then, on $N \backslash \overline{B\left(A, \varepsilon_{1}\right)}, h_{1}$ takes value 0 and $g_{1}$ takes value $y^{\prime}$. Thus $g_{1}(x)=\phi_{A}^{1} \circ h_{1}(x)$, both on $\overline{B\left(A, \varepsilon_{1}\right)}$ and on $N \backslash \overline{B\left(A, \varepsilon_{1}\right)}$, and so on $N$. Hence $g_{1}$ is again continuous at $x_{0}$.

In particular, $g_{1}$ is a continuous function on $X$, with

$$
g_{1}(x)= \begin{cases}g_{0}(x) & \text { on } E_{1}=X \backslash \bigcup\left\{B\left(A, \varepsilon_{1}\right): A \in \mathcal{D}_{1}\right\}, \\ y_{A} & \text { on } \overline{B\left(A, \varepsilon_{1} / 2\right)} \text { for each } A \in \mathcal{D}_{1} .\end{cases}
$$

Now suppose that for some $n, 1 \leq n<p$, we have defined continuous functions $g_{1}, \ldots, g_{n}$ on $X$ so that

$$
g_{n}(x)= \begin{cases}g_{n-1}(x) & \text { on } E_{n}=X \backslash \bigcup\left\{B\left(A, \varepsilon_{n}\right): A \in \mathcal{D}_{n}\right\} \\ y_{A} & \text { on } \overline{B\left(A, \varepsilon_{n} / 2\right)} \text { for each } A \in \mathcal{D}_{n} .\end{cases}
$$

Write

$$
\begin{aligned}
& E_{n+1}=X \backslash \bigcup\left\{B\left(A, \varepsilon_{n+1}\right): A \in \mathcal{D}_{n+1}\right\}, \\
& D_{n+1}=\bigcup\left\{\overline{B\left(A, \varepsilon_{n+1} / 2\right)}: A \in \mathcal{D}_{n+1}\right\} .
\end{aligned}
$$

Since the family $\left\{B\left(A, \varepsilon_{n+1}\right): A \in \mathcal{D}_{n+1}\right\}$ is discrete, $E_{n+1}$ and $D_{n+1}$ are disjoint closed sets. Hence

$$
h_{n+1}(x)=\frac{d\left(x, E_{n+1}\right)}{d\left(x, D_{n+1}\right)+d\left(x, E_{n+1}\right)}
$$

is a continuous function on $X$ taking value 1 on $D_{n+1}$ and 0 on $E_{n+1}$. Take

$$
g_{n+1}(x)= \begin{cases}g_{n}(x) & \text { on } E_{n+1}, \\ y_{A} & \text { on } \overline{B\left(A, \varepsilon_{n+1} / 2\right)} \text { for } A \in \mathcal{D}_{n+1}, \\ \phi_{A}^{n+1} \circ h_{n+1} & \text { on } B\left(A, \varepsilon_{n+1}\right) \backslash \overline{B\left(A, \varepsilon_{n+1} / 2\right)} \text { for } A \in \mathcal{D}_{n+1} .\end{cases}
$$


Since we have assigned values to $g_{n+1}$ on a family of disjoint sets with union $X$, the function $g_{n+1}$ is well-defined. We verify that it is continuous on $X$. Let $x_{0}$ be any point of $X$. If $x_{0}$ belongs to none of the sets of the discrete family $\left\{\overline{B\left(A, \varepsilon_{n+1}\right)}: A \in \mathcal{D}_{n+1}\right\}$ of closed sets we can choose a neighbourhood $N$ of $x_{0}$ that meets none of these sets. Then $g_{n+1} \underline{(x)=g_{n}(x)}$ on $N$ and so $g_{n+1}$ is continuous at $x_{0}$. Suppose now that $x_{0} \in \overline{B\left(A, \varepsilon_{n+1}\right)}$ for some $A \in \mathcal{D}_{n+1}$. Then $x_{0} \in B\left(C, \varepsilon_{n} / 2\right)$ for just one $C$ in $\mathcal{D}_{n}$. Now we can take $N$ to be a neighbourhood of $x_{0}$ contained in $B\left(C, \varepsilon_{n} / 2\right)$ that necessarily meets $\overline{B\left(A, \varepsilon_{n+1}\right)}$ but meets no set $\overline{B\left(A^{\prime}, \varepsilon_{n+1}\right)}$ with $A^{\prime} \in \mathcal{D}_{n+1}$, $A^{\prime} \neq A$. Now on $N \backslash B\left(A, \varepsilon_{n+1}\right) \subset E_{n+1}, h_{n+1}$ takes value 0 and $g_{n+1}(x)=$ $g_{n}(x)=y_{C}$, since $N \subset B\left(C, \varepsilon_{n} / 2\right)$, and so

$$
g_{n+1}(x)=\phi_{A}^{n+1} \circ h_{n+1}(x) .
$$

The same is true on $N \cap B\left(A, \varepsilon_{n+1}\right) \backslash \overline{B\left(A, \varepsilon_{n+1} / 2\right)}$. Further, on $\overline{B\left(A, \varepsilon_{n+1} / 2\right)}, h_{n+1}(x)$ takes value 1 and $g_{n+1}(x)$ takes value $y_{A}$ so that (*) holds again. Thus it holds on $N$ and $g_{n+1}$ is continuous at $x_{0}$. It follows, in particular, that whenever $g_{n+1}(x) \neq g_{n}(x)$ we have

$$
g_{n+1}(x)=\phi_{A}^{n+1} \circ h_{n+1}(x) \text { and } g_{n}(x)=y_{C} .
$$

Since the arc in $Y$ given by $\phi_{A}^{n+1}(t), 0 \leq t \leq 1$, is of diameter at most $2^{-n}$, we have

for all $x$ in $X$.

$$
d\left(g_{n+1}(x), g_{n}(x)\right) \leq 2^{-n},
$$

Proceeding inductively in this way we define continuous functions $g_{0}, g_{1}$, $\ldots, g_{p}=g$ on $X$ satisfying

$$
g_{n}(x)=y_{A} \quad \text { for } x \in A \in \mathcal{D}_{n} \text { and } 1 \leq n \leq p,
$$

and

$$
d\left(g_{n}(x), g_{n+1}(x)\right) \leq 2^{-n} \quad \text { for } x \in X \text { and } 1 \leq n<p .
$$

Thus

$$
d\left(g(x), g_{n}(x)\right) \leq 2^{-n}+2^{-n-1}+\ldots+2^{-p+1} \leq 2^{-n+1}
$$

for $x \in X$ and $1 \leq n \leq p$. Now, if $x \in A \in \mathcal{D}_{n}, 1 \leq n \leq p$, we have

$$
d\left(g(x), y_{A}\right)=d\left(g(x), g_{n}(x)\right) \leq 2^{-n+1} .
$$

Since $y_{A} \in f(A)$ and any two points of $f(A)$ can be joined by an arc of diameter less than $2^{-n}$, we have

$$
d\left(f(x), y_{A}\right) \leq 2^{-n} .
$$

Hence $d(f(x), g(x)) \leq 2^{-n+2}$ as required.

THEOREM 1. Let $X$ be a metric space and let $Y$ be an arcwise connected and locally arcwise connected metric space. Then $\mathcal{B}_{1}(X, Y) \cap \Sigma(X, Y)=$ $\mathcal{B} a_{1}(X, Y)$. 
Proof. Every first Baire class function is $\sigma$-discrete and of first Borel class, so let $f \in \mathcal{B} o_{1}(X, Y) \cap \Sigma(X, Y)$. We shall define a sequence of continuous functions, converging pointwise to $f$.

Using local arcwise connectedness find, for each $y \in Y$ and each $n$, an open neighbourhood $U_{n}(y)$ of $y$ such that any two points of $U_{n}(y)$ can be joined with an arc of diameter at most $2^{-n}$.

Since $f$ is $\sigma$-discrete and of first Borel class, it has a $\sigma$-discrete closed base, which we will denote by $\mathcal{A}$. For each $n$ define $\mathcal{A}_{n}=\{A \in \mathcal{A}: \exists y \in Y$ $\left.f(A) \subseteq U_{n}(y)\right\}$. Note that $\bigcup \mathcal{A}_{n}=X$ for each $n$.

For each $n$ apply Lemma 1 to the family $\mathcal{A}_{n}$ to find families $\mathcal{B}_{n}^{p}$ of closed sets satisfying:

- each member of $\mathcal{B}_{n}^{p}$ is contained in some member of $\left\{f^{-1}\left(U_{n}(y)\right)\right.$ : $y \in Y\}$

- each member of $\mathcal{B}_{n}^{p}$ is contained in some member of $\mathcal{B}_{n}^{p+1}$;

- $\mathcal{B}_{n}^{p}$ is uniformly discrete for all $p$; and

- $\bigcup_{p} \mathcal{B}_{n}^{p}$ is a cover of $X$ (for all $n$ ).

We shall use these families to construct a new set of families of closed sets by defining, for all $p$ and $n$,

$$
\mathcal{D}_{n}^{p}=\left\{A_{1} \cap \ldots \cap A_{n}: A_{m} \in \mathcal{B}_{m}^{p} \text { for all } m \leq n\right\} .
$$

Note that $\mathcal{D}_{n}^{p}$ is uniformly discrete, that each member of $\mathcal{D}_{n+1}^{p}$ is contained in some member of $\mathcal{D}_{n}^{p}$ and that $\bigcup_{p} \mathcal{D}_{n}^{p}$ covers $X$ for all $n$. For each $p$ apply Lemma 2 to $f$ and the families $\mathcal{D}_{n}^{p}, n \leq p$, to get continuous functions $g_{p}$ : $X \rightarrow Y$ such that, whenever $x \in \bigcup_{n \leq p} \mathcal{D}_{n}^{p}$ and $p \geq n$, then $d\left(f(x), g_{p}(x)\right)$ $\leq 2^{-n+2}$.

The sequence $\left\{g_{p}\right\}$ converges pointwise to $f$. To see this let $\varepsilon>0$ and $x \in X$ be given. Find $n$ such that $2^{-n+2} \leq \varepsilon$ and find $p \geq n$ such that $x \in \bigcup \mathcal{D}_{n}^{p}$. Then $d\left(f(x), g_{q}(x)\right) \leq \varepsilon$ for all $q \geq p$.

We now show that the converse of Theorem 1 is true when $X$ is the unit interval $I$ and $Y$ is complete. This will be done through a series of lemmas.

Lemma 3. Assume $\mathcal{B} a_{1}(I, Y)=\mathcal{B} o_{1}(I, Y)$ where $Y$ is a metric space. Then for all $\varepsilon>0$ and all $y \in Y$ there exists an open neighbourhood $U(y)$ of $y$ such that, for all nonempty open subsets $U_{0}, U_{1}$ of $U(y)$, there exists $\phi \in \mathcal{B} a_{0}(I, Y)$ with $\phi(i) \in U_{i}$ for $i=0,1$ and $\operatorname{diam} \phi(I) \leq \varepsilon$.

Proof. Assume the statement is not true. Then we can find $\varepsilon>0$ and $y \in Y$ such that, in all open balls $B\left(y, 2^{-n} \varepsilon\right)$, there exist two nonempty open sets $U(n, 0)$ and $U(n, 1)$ with the property that every $\operatorname{arc} \phi \in \mathcal{B} a_{0}(I, Y)$ with $\phi(i) \in U(n, i)$ for $i=0,1$ satisfies $\operatorname{diam} \phi(I)>\varepsilon$. For each $n$ and $i$ pick a point $y(n, i) \in U(n, i)$. 
Let $\alpha: \mathbb{N} \times\{0,1\} \rightarrow \mathbb{Q}$ be a 1-1 map such that for any open interval $\left(t_{0}, t_{1}\right) \subset I$ there exists $n$ with $t_{0}<\alpha(n, 0)<\alpha(n, 1)<t_{1}$. Define the function $f: I \rightarrow Y$ by

$$
f(t)= \begin{cases}y & \text { if } t \in I \backslash \alpha(\mathbb{N} \times\{0,1\}), \\ y(n, i) & \text { if } t=\alpha(n, i),(n, i) \in \mathbb{N} \times\{0,1\} .\end{cases}
$$

Note that $f(I)$ is contained in $B(y, \varepsilon / 2)$. Since the $y(n, i)$ converge to $y$, the function $f$ is of the first Borel class, and so, by assumption, there exists a sequence $\left\{\phi_{k}\right\} \subset \mathcal{B} a_{0}(I, Y)$ converging pointwise to $f$.

Define for each $m$ the set $H_{m}=\bigcup_{k>m} \phi_{k}^{-1}(Y \backslash \overline{B(y, \varepsilon / 2)})$. Then each $H_{m}$ is open and dense. To see this let $0 \leq t_{0}<t_{1} \leq 1$ and find $n$ such that $t_{0}<\alpha(n, 0)<\alpha(n, 1)<t_{1}$. The sequence $\left\{\phi_{k}\right\}$ converges pointwise to $f$, so we can find $k \geq m$ so large that $\phi_{k}(\alpha(n, i)) \in U(n, i)$ for $i=0,1$. By the definition of $U(n, i), i=0,1$, we have diam $\phi_{k}(\alpha(n, 0), \alpha(n, 1))>\varepsilon$. So there exists $t_{2} \in(\alpha(n, 0), \alpha(n, 1))$ such that $\phi_{k}\left(t_{2}\right) \in Y \backslash \overline{B(y, \varepsilon / 2)}$.

Using the Baire category theorem we get $\bigcap_{m} H_{m} \neq 0$, which contradicts $\bigcap_{m} H_{m} \subseteq f^{-1}(Y \backslash B(y, \varepsilon / 2))=\emptyset$.

Lemma 4. Let $Y$ be complete. Then $Y$ is locally arcwise connected if and only if for all $\varepsilon>0$ and for all $y \in Y$ there exists an open neighbourhood $U$ of $y$ such that for all nonempty open sets $U_{0}, U_{1} \subset U$ there exists an arc of diameter less than $\varepsilon$ starting in $U_{0}$ and ending in $U_{1}$.

Proof. The "only if" part is trivial. To show the "if" part let $\varepsilon>0$, $y \in Y$ and let $U$ be an open neighbourhood of $y$ such that any two nonempty open sets $U_{0}, U_{1}$ contained in $U$ can be joined with an arc of diameter less than $\varepsilon / 3$ starting in $U_{0}$ and ending in $U_{1}$. Let $V \subset Y$ be obtained from $U$ by adding to $U$ all arcs intersecting $U$ and having diameter less than $\varepsilon / 3$. We prove that $V$ is connected. Indeed, if $V=G_{0} \cup G_{1}$, where $G_{0}$ and $G_{1}$ are nonempty, disjoint relatively open subsets of $V$, then each arc in $V$ lies in either $G_{0}$ or in $G_{1}$. Since each point of $V \backslash U$ lies, by definition, on an arc in $V$ that intersects $U$, it follows that $U \cap G_{0} \neq \emptyset$ and $U \cap G_{1} \neq \emptyset$. Since $U \cap G_{0}$ and $U \cap G_{1}$ are open sets, we can find an arc of diameter less than $\varepsilon / 3$ joining $U \cap G_{0}$ and $U \cap G_{1}$. By definition this arc lies in $V$, which is impossible since it intersects both $G_{0}$ and $G_{1}$. Finally, we observe that $\operatorname{diam} U \leq \varepsilon / 3$, hence $\operatorname{diam} V \leq 2 \varepsilon / 3<\varepsilon$, which shows that $V$ is a connected neighbourhood of $y$ of diameter less than $\varepsilon$.

Now [11, Theorem 1, p. 254] shows that $Y$ is locally arcwise connected.

Lemma 5. Let $Y$ be complete and assume that $\mathcal{B} a_{1}(I, Y)=\mathcal{B} o_{1}(I, Y)$. Then $Y$ is arcwise connected.

Proof. Let $y_{0}, y_{1} \in Y$. We shall define an arc joining these two points. Use Lemmas 3 and 4 to find open neighbourhoods $U_{0}, U_{1}$ of $y_{0}$ and $y_{1}$, 
respectively, such that any pair of points of $U_{0}$ can be joined with an arc, and likewise for $U_{1}$.

The function

$$
f(t)= \begin{cases}y_{0} & \text { if } t=0, \\ y_{1} & \text { if } t>0,\end{cases}
$$

from $I$ to $Y$ is of first Borel class. By assumption $f$ is then of first Baire class, so there exists an $\operatorname{arc} \phi \in \mathcal{B} a_{0}(I, Y)$ with $\phi(i) \in U_{i}$ for $i=0,1$. Join $y_{0}$ to $\phi(0)$ and $\phi(1)$ to $y_{1}$ with arcs. Then these three arcs together join $y_{0}$ and $y_{1}$.

TheOREM 2. Let $Y$ be complete metric. Then the following three statements are equivalent:

(i) $Y$ is connected and locally connected;

(ii) $\mathcal{B} a_{1}(I, Y)=\mathcal{B} o_{1}(I, Y)$; and

(iii) $\mathcal{B} a_{1}(X, Y)=\mathcal{B} o_{1}(X, Y) \cap \Sigma(X, Y)$ for all metric spaces $X$.

Rem a rk. The implication: $Y$ is arcwise connected and locally arcwise connected $\Rightarrow$ (iii) is the statement of Theorem 1 which does not assume that $Y$ is complete.

P r o of. Theorem 1, p. 254 in [11] shows that (i) implies that $Y$ is locally arcwise connected. This again implies that $Y$ is arcwise connected. To see this let $y \in Y$ and let $U$ be the union of all arcs going through $y$. Since $Y$ is locally arcwise connected $U$ is a nonempty clopen set. Since $Y$ is connected we conclude that $U$ equals $Y$, and hence that $Y$ is arcwise connected. Now, to obtain the conclusion of the theorem, combine Lemmas 3-5 and Theorem 1 and note that every function from $I$ to $Y$ is $\sigma$-discrete.

Note that the proofs of Lemmas 3 and 5 still work when $I$ is replaced by a metric space $X$ containing a homeomorphic copy of $I$. Therefore we immediately get the following corollary.

COROLlary. Let $X$ be a metric space that contains a homeomorphic copy of $I$. Let $Y$ be a complete metric space. Then $\mathcal{B} a_{1}(X, Y)=\mathcal{B o}_{1}(X, Y) \cap$ $\Sigma(X, Y)$ if and only if $Y$ is locally connected and connected.

Rem ark. The remark after Theorem 2 applies here as well.

Next we shall look at some examples. The first will show that Lemmas 45 fail to be true in general if $Y$ is not complete, and that it is not enough to assume that $Y$ is a $K_{\sigma}$-set in a complete separable metric space to obtain the conclusions of the lemmas.

We will be working in the Hilbert cube $I^{\mathbb{N}}$, equipped with the complete metric $d\left(\left(t_{i}\right),\left(s_{i}\right)\right)=\sum_{i} 2^{-i}\left|t_{i}-s_{i}\right|$, and we will let $\pi_{i}$ be the projection on the $i$ th coordinate.

EXAMPLE 1. There exists a $K_{\sigma}$-set $Y \subset I^{\mathbb{N}}$ such that: 
(i) $\mathcal{B} o_{1}(X, Y)=\mathcal{B} a_{1}(X, Y)$ for all metric spaces $X$; and

(ii) $Y$ is not locally arcwise connected nor arcwise connected.

For all $p$ let $Y_{p}=\left\{\left(t_{i}\right) \in I^{\mathbb{N}}: t_{p}=1, t_{i}=0 \forall i>p\right\}$. Then $\left\{Y_{p}: p \in \mathbb{N}\right\}$ is a countable and disjoint collection of compact sets. Define $Y=\bigcup_{p} Y_{p}$.

(i) Let $f \in \mathcal{B}_{1}(X, Y)$. Then $\pi_{k} \circ f \in \mathcal{B}_{o_{1}}(X, I)$ for each $k$, since the projection is continuous. By Theorem 1 there exist sequences $\left\{g_{k}^{m}\right\} \subseteq \mathcal{B} a_{0}(X, I)$ of continuous functions converging pointwise to $\pi_{k} \circ f$ for each $k$. Now $g^{m}=\left(g_{1}^{m}, g_{2}^{m}, \ldots, g_{m}^{m}, 1,0,0, \ldots\right)$ is a continuous function from $X$ to $Y_{m+1}$ and $\left\{g^{m}\right\}$ converges pointwise to $f$.

(ii) Let $\phi: I \rightarrow Y$ be an arc. Then $\phi^{-1}\left(Y_{p}\right) \neq \emptyset$ for exactly one $p$ for otherwise we could write $I$ as a disjoint union of countably many but at least two nonempty closed sets, which is impossible by the Sierpiński Theorem in [3, p. 440]. This shows that $Y$ is not arcwise connected. In fact, it also shows that $Y$ is not locally arcwise connected, for any open nonempty subset of $Y$ intersects infinitely many of the sets $\left\{Y_{p}: p \in \mathbb{N}\right\}$ and, as we have just seen, a pair of points that do not lie in one of these sets cannot be joined with an arc.

If a metric space $Y$ satisfies $\mathcal{B} a_{1}(I, Y)=\mathcal{B} o_{1}(I, Y)$, then $Y$ must have the following two properties which we call $\left(\mathrm{P}^{1}\right)$ and $\left(\mathrm{P}^{2}\right)$.

$\left(\mathrm{P}^{1}\right) \quad$ For all finite sequences of nonempty open sets $U_{1}, \ldots, U_{n} \subset Y$ there exists an $\operatorname{arc} \phi \in \mathcal{B} a_{0}(I, Y)$ such that $\phi^{-1}\left(U_{i}\right) \neq \emptyset$ for all $i \leq n$.

$\left(\mathrm{P}^{2}\right) \quad$ For all $\varepsilon>0$ and all $y \in Y$ there exists an open neighbourhood $U$ of $y$ such that, for all finite sequences of nonempty open sets $U_{1}, \ldots, U_{n} \subset$ $U$, there exists an $\operatorname{arc} \phi \in \mathcal{B} a_{0}(I, Y)$ such that $\phi^{-1}\left(U_{i}\right) \neq \emptyset$ for all $i \leq n$ and such that $\operatorname{diam} \phi(I) \leq \varepsilon$.

That $\left(\mathrm{P}^{1}\right)$ must hold can be shown with an argument similar to that in the proof of Lemma 5 , and that $\left(\mathrm{P}^{2}\right)$ must hold can be shown along the lines of the proof of Lemma 3.

Conversely, if a complete space $Y$ satisfied $\left(\mathrm{P}^{1}\right)$ and $\left(\mathrm{P}^{2}\right)$ then by Lemma 4 it would be arcwise connected and locally arcwise connected. If $Y$ were also separable, then we would have $\mathcal{B} a_{1}(X, Y)=\mathcal{B} o_{1}(X, Y)$ for all metric spaces $X$. The next example shows that this conclusion fails when $Y$ is not complete. Indeed, it shows that the conclusion fails for a space that satisfies $\left(\mathrm{P}^{1}\right)$ and $\left(\mathrm{P}^{2}\right)$ and is a $K_{\sigma}$-set in a complete separable metric space.

EXAMPLE 2 . There exists a $K_{\sigma}$-subset $Y$ of $I^{\mathbb{N}}$ satisfying $\left(\mathrm{P}^{1}\right)$ and $\left(\mathrm{P}^{2}\right)$ but where $\mathcal{B} a_{1}(I, Y) \neq \mathcal{B} o_{1}(I, Y)$.

We shall construct the set $Y$. Consider the following subsets of $I^{\mathbb{N}}$ :

$$
\begin{aligned}
& Y_{p}^{1}=\left\{\left(t_{i}\right) \in I^{\mathbb{N}}: t_{p}=1, t_{i}=0 \forall i>p\right\}, \\
& Y_{p}^{2}=\left\{\left(t_{i}\right) \in I^{\mathbb{N}}: t_{p}=1 / 2, t_{i}=0 \forall i>p\right\} .
\end{aligned}
$$


These sets are disjoint. To see this assume that $Y_{p_{1}}^{j_{1}} \cap Y_{p_{2}}^{j_{2}}$ is nonempty. We cannot have $p_{1} \neq p_{2}$ because of the restriction on the last nonzero coordinate. For the same reason we cannot have $j_{1} \neq j_{2}$.

The space $I^{\mathbb{N}}$ has a countable base of sets of the form

$$
O_{1} \times \ldots \times O_{k} \times I \times I \times \ldots
$$

with $O_{1}, \ldots, O_{k}$ nonempty open subsets of $I$, so the set of all finite sequences of sets from this base is countable. We denote it by

$$
\left\{\left(U_{1}^{i}, U_{2}^{i}, \ldots, U_{\sigma(i)}^{i}\right): i \in \mathbb{N}\right\} .
$$

Note that the function $\sigma: \mathbb{N} \rightarrow \mathbb{N}$ determines the length of each of the finite sequences.

For each $i$ there exists a $q(i)$ such that $\pi_{q}\left(U_{j}^{i}\right)=I$ for all $q \geq q(i)$ and all $j \leq \sigma(i)$. Let $p(1)=\max \{q(1), 3\}$ and define inductively $p(i+1)=$ $\max \{q(i+1), p(i)+1\}$. Then $\{p(i)\}$ is a strictly increasing sequence of integers, all greater than 3 , and such that $U_{j}^{i} \cap Y_{p(i)}^{1} \neq \emptyset$ and $U_{j}^{i} \cap Y_{p(i)}^{2} \neq \emptyset$ for all $i$ and $j \leq \sigma(i)$. To see this fix $i$ and $j$ and write $U_{j}^{i}=V \times I^{\mathbb{N}}$ where $V$ is an open subset of $I^{p(i)-1}$. Let $\left(t_{1}, \ldots, t_{p(i)-1}\right) \in V$. Then $\left(t_{1}, \ldots, t_{p(i)-1}, 1,0,0, \ldots\right) \in U_{j}^{i} \cap Y_{p(i)}^{1}$ and $\left(t_{1}, \ldots, t_{p(i)-1}, 1 / 2,0,0, \ldots\right) \in$ $U_{j}^{i} \cap Y_{p(i)}^{2}$.

For each $i$ and $j \leq \sigma(i)$ pick a point $u_{j}^{i} \in U_{j}^{i} \cap Y_{p(i)}^{1}$. For all $i$ let $y^{i}=(0,1,0,0, \ldots, 0,1,0,0, \ldots)$, where the second 1 is the $p(i)$ th coordinate. Note that the distance between $y^{i}$ and $y=(0,1,0,0, \ldots)$ is $2^{-p(i)}$, and that $2^{-p(i)} \leq 2^{-3}$ for all $i$. by

For each $i$ we define a continuous piecewise linear function $\phi_{i}^{1}: I \rightarrow Y_{p(i)}^{1}$

$$
\phi_{i}^{1}(t)=\left\{\begin{array}{c}
y^{i} \quad \text { if } t \leq \frac{1}{2 \sigma(i)}, \\
y^{i}+\left(t-\frac{j}{\sigma(i)}+\frac{1}{2 \sigma(i)}\right) 2 \sigma(i)\left(u_{j}^{i}-y^{i}\right) \\
\text { if } \frac{j}{\sigma(i)}-\frac{1}{2 \sigma(i)}<t \leq \frac{j}{\sigma(i)}, j \leq \sigma(i)-1, \\
u_{j}^{i}+\left(t-\frac{j}{\sigma(i)}\right) 2 \sigma(i)\left(y^{i}-u_{j}^{i}\right) \quad \text { if } \frac{j}{\sigma(i)}<t \leq \frac{j}{\sigma(i)}+\frac{1}{2 \sigma(i)} .
\end{array}\right.
$$

Note that $Y_{p(i)}^{1}$ is convex and that $\phi_{i}^{1}(I)$ is the union of $\sigma(i)$ straight-line segments joining $y^{i}$ to the points $u_{j}^{i}$.

Let $N=\left\{i \in \mathbb{N}: \operatorname{diam}\left(\bigcup_{j \leq \sigma(i)} U_{j}^{i}\right) \leq 1 / 2\right\}$. For each $i \in N$ let $\phi_{i}^{2}$ : $I \rightarrow Y_{p(i)}^{2}$ be a piecewise linear continuous function satisfying:

- $\phi_{i}^{2}(I) \cap U_{j}^{i} \neq \emptyset \forall j \leq \sigma(i)$; and

- $\operatorname{diam} \phi_{i}^{2}(I) \leq \operatorname{diam}\left(\bigcup_{j \leq \sigma(i)} U_{j}^{i}\right)$. 
We can construct these functions by the method used to construct the functions $\phi_{i}^{1}$.

The space providing the example is defined by

$$
Y=Y_{2}^{1} \cup Y_{2}^{2} \cup \bigcup_{i \in \mathbb{N}} \phi_{i}^{1}(I) \cup \bigcup_{i \in N} \phi_{i}^{2}(I) .
$$

Note the following points:

- $Y$ is a $K_{\sigma}$-set;

- any arc $\psi(I) \subset Y$ must be contained in one of the disjoint sets of which $Y$ is the union;

- the subset $\left\{\phi_{i}^{1}(I)\right\}$ of $Y$ ensures that $Y$ satisfies $\left(\mathrm{P}^{1}\right)$; and

- the subset $\left\{\phi_{i}^{2}(I): i \in N\right\}$ of $Y$ ensures that $Y$ satisfies $\left(\mathrm{P}^{2}\right)$.

Let us assume that $\mathcal{B} a_{1}(I, Y)=\mathcal{B} o_{1}(I, Y)$ and seek a contradiction to prove that $Y$ is indeed the example. Define the first Borel class function $f: I \rightarrow Y$ by

$$
f(t)= \begin{cases}(t, 1 / 2,0,0, \ldots) & \text { if } t<1, \\ (1,1,0,0, \ldots) & \text { if } t=1 .\end{cases}
$$

Then, by assumption, we can find a sequence $\left\{\psi_{n}\right\} \subset \mathcal{B} a_{0}(I, Y)$ converging pointwise to $f$. For $n$ sufficiently large the points $\psi_{n}(0)$ and $\psi_{n}(1)$ are near $(t, 1 / 2,0,0, \ldots)$ and $(1,1,0,0, \ldots)$. We may assume that $\psi_{n}(I) \subseteq \bigcup_{i \in \mathbb{N}} \phi_{i}^{1}(I)$ for all $n$, for all arcs contained in one of the sets $\phi_{i}^{2}(I), i \in \mathbb{N}$, would have too small a diameter. In fact, $\psi_{n}(I) \subseteq \phi_{i}^{n}(I)$ for some $i=i(n)$.

Let $y=(0,1,0,0, \ldots)$, which is in $Y_{2}^{1}$, and for each $m$ define $H_{m}=$ $\bigcup_{n \geq m} \psi_{n}^{-1}(B(y, 1 / 4))$. Then clearly $H_{m}$ is open and $y^{i} \in B(y, 1 / 4)$ for all $i$. To see that $H_{m}$ is also dense in $[1 / 2,1]$ let $a$ and $b$ be such that $1 / 2<a<b<1$. For $\varepsilon$ sufficiently small there is no line that intersects $B(f(a), \varepsilon), B(f(b), \varepsilon)$ and $\{y\} \cup \bigcup_{i}\left\{y^{i}\right\}$. However, for large $n$ we have $\psi_{n}(a) \in B(f(a), \varepsilon)$ and $\psi_{n}(b) \in B(f(b), \varepsilon)$. But $\psi_{n}(I) \subseteq \phi_{i}^{1}(I)$ for some $i$, so, since $\psi_{n}((a, b))$ cannot be contained in one of the linear segments of $\phi_{i}^{1}(I)$, we see that $\psi_{n}((a, b))$ cannot lie on just one of the line segments making up $\phi_{i}^{1}(I)$. Therefore $y^{i} \in \psi_{n}((a, b))$ and so $\psi_{n}((a, b)) \cap B(y, 1 / 4) \neq \emptyset$.

Hence each $H_{m}$ is dense and open in $[1 / 2,1]$ and so $\bigcap_{m} H_{m} \cap[1 / 2,1] \neq$ $\emptyset$ by the Baire category theorem, which contradicts $\bigcap_{m} H_{m} \cap[1 / 2,1] \subseteq$ $f^{-1}(\overline{B(y, 1 / 4)})=\emptyset$. We conclude that $\mathcal{B} a_{1}(I, Y) \neq \mathcal{B} o_{1}(I, Y)$ as required.

EXAmple 3. Consider the subset

$$
M=\{(x, \sin (\pi / x)): 0<x \leq 1\} \cup\{0\} \times[-1,2] \cup[0,1] \times\{2\} \cup\{1\} \times[0,2]
$$

of $\mathbb{R}^{2}$. The space $M$ is metric, separable, compact, complete, arcwise connected and a continuous image of the real line. But $M$ is not locally arcwise connected and so, by Theorem 2 , we have $\mathcal{B} a_{1}(I, M) \neq \mathcal{B} o_{1}(I, M)$. 


\section{Second Baire class functions}

LemMa 6. If $\bigcup_{\alpha<\omega_{1}} \mathcal{B} a_{\alpha}(X, Y)=\bigcup_{\alpha<\omega_{1}} \mathcal{B} o_{\alpha}(X, Y)$, then, for all $q \in \mathbb{N}$, for all sequences $U_{1}, \ldots, U_{q}$ of nonempty open subsets of $Y$ and for all sequences $x_{1}, \ldots, x_{q}$ of distinct points of $X$, there exists a continuous function $\phi: X \rightarrow Y$ with $\phi\left(x_{i}\right) \in U_{i}$ for all $i \leq q$.

Proof. Let $U_{1}, \ldots, U_{q}$ be a sequence of nonempty open subsets of $Y$ and $x_{1}, \ldots, x_{q}$ be a sequence of distinct points of $X$. Pick for each $i \leq q$ a point $y_{i} \in U_{i}$. Define the $\sigma$-discrete first Borel class function

$$
f(x)= \begin{cases}y_{i} & \text { if } x=x_{i}, q \geq i>1 \\ y_{1} & \text { otherwise }\end{cases}
$$

Then by assumption $f$ is a Baire class $\alpha$ function, for some $\alpha<\omega_{1}$, and $f\left(x_{i}\right) \in U_{i}$ for all $i \leq q$.

Observe that, whenever a noncontinuous function $g$ of Baire class $\beta$ satisfies $g\left(x_{i}\right) \in U_{i}$ for all $i \leq q$, then there exists $\eta<\beta$ and a function $h$ of Baire class $\eta$ such that $h\left(x_{i}\right) \in U_{i}$ for all $i \leq q$.

Apply this observation to $f$ to get a function $f_{1}$ of strictly lower Baire class than that of $f$, with $f_{1}\left(x_{i}\right) \in U_{i}$ for all $i \leq q$. If $f_{1}$ is continuous, then $f_{1}$ satisfies the conclusion of the lemma. If $f_{1}$ is not continuous, we apply our observation to $f_{1}$ to get a function $f_{2}$ of strictly lower Baire class than that of $f_{1}$. We repeat this process until it halts. The process will indeed halt, since a strictly decreasing sequence of ordinals can only be finite. The function we have when the process halts satisfies the conclusion of the lemma.

LEMMA 7. If $Y$ is separable, metric and $X$ is metric such that for all $q \in \mathbb{N}$, for all sequences $F_{1}, \ldots, F_{q}$ of disjoint closed subsets of $X$ and for all sequences $U_{1}, \ldots, U_{q}$ of nonempty open subsets of $Y$, there exists a continuous function $\phi: X \rightarrow Y$ with $\phi\left(F_{i}\right) \subseteq U_{i}$ for all $i \leq q$, then $\mathcal{B} a_{2}(X, Y)=\mathcal{B o}_{2}(X, Y)$.

Pro of. We will first show that all first Borel class functions from $X$ to $Y$ with finite range are of first Baire class. So let $f: X \rightarrow Y$ be a first Borel class function with finite range. Let $\left\{y_{i}: i \leq q\right\}$ be the finite range, and let, for each $m,\left\{F_{i m}: i \leq q\right\}$ be a disjoint family of closed sets such that $f^{-1}\left(y_{i}\right)=\bigcup_{m} F_{i m}$ for every $i \leq q$. By hypothesis we can find continuous functions $\phi_{n}: I \rightarrow Y, n \in \mathbb{N}$, so that $\phi_{n}\left(\bigcup_{m \leq n} F_{i m}\right) \subseteq B\left(y_{i}, 2^{-n}\right)$ for all $i \leq q$. Then the sequence $\left\{\phi_{n}\right\}$ converges pointwise to $f$.

Now let $h: X \rightarrow Y$ be of second Borel class. We shall show that $h$ is of second Baire class. In [10, p. 389-391], it is proved that all second class Borel functions from a metric space to a separable metric space are pointwise limits of first Borel class functions with finite range. By the argument above, all these first Borel class functions are of first Baire class. Thus $h$ is of second Baire class. 
TheOREM 3. Let $Y$ be a separable metric space. Let $X$ be a metric space containing a homeomorphic copy of the unit interval. The following statements are equivalent:

(i) $\mathcal{B} a_{2}(I, Y)=\mathcal{B} o_{2}(I, Y)$;

(ii) For all $q \in \mathbb{N}$, for all sequences $x_{1}, \ldots, x_{q}$ of distinct points of $I$ and for all sequences $U_{1}, \ldots, U_{q}$ of nonempty open subsets of $Y$, there exists a continuous function $\phi: I \rightarrow Y$ with $\phi\left(x_{i}\right) \in U_{i}$ for all $i \leq q$;

(iii) For all $q \in \mathbb{N}$, for all sequences $F_{1}, \ldots, F_{q}$ of disjoint closed subsets of $X$ and for all sequences $U_{1}, \ldots, U_{q}$ of nonempty open subsets of $Y$, there exists a continuous function $\phi: X \rightarrow Y$ with $\phi\left(F_{i}\right) \subseteq U_{i}$ for all $i \leq q$;

(iv) $\mathcal{B} a_{2}(X, Y)=\mathcal{B o}_{2}(X, Y)$;

(v) For each finite (countable infinite) ordinal $\alpha \geq 2$ we have $\mathcal{B} a_{\alpha}(X, Y)$ equal to $\mathcal{B} o_{\alpha}(X, Y)\left(\mathcal{B} o_{\alpha+1}(X, Y)\right)$; and

(vi) $\bigcup_{\alpha<\omega_{1}} \mathcal{B} a_{\alpha}(X, Y)=\bigcup_{\alpha<\omega_{1}} \mathcal{B} o_{\alpha}(X, Y)$.

P r o of. (i) $\Rightarrow$ (ii) follows from the argument of Lemma 6 .

(ii) $\Rightarrow$ (iii). By Tietze's theorem let $f: X \rightarrow I$ be a continuous extension of the function defined by $f(x)=i / q \Leftrightarrow x \in F_{i}$. By (ii) we can find a continuous function $\phi: I \rightarrow Y$ with $\phi(i / q) \in U_{i}$ for all $i \leq q$. The function $\phi \circ f$ satisfies the condition in (iii).

(iii) $\Rightarrow$ (iv) is Lemma 7 .

(iv) $\Rightarrow$ (v) follows from the remark in Section 1, or from Banach's theorem in $[10$, p. 394].

$(\mathrm{v}) \Rightarrow(\mathrm{vi})$ is trivial.

(vi) $\Rightarrow(\mathrm{i})$. By Lemma 6 we see that $I$ satisfies (ii), since $I \subseteq X$. We have already noted that (ii) implies (iv) so $I$ satisfies (iv), which is the statement of (i).

Let $X$ be a topological space and let $Y$ be a metric space. Every $\sigma$ discrete second Borel class function from $X$ to $Y$ is, by [6, Theorem 5], the pointwise limit of a sequence of $\sigma$-discrete first Borel class functions, each of which has discrete range.

Let us assume that $X$ and $Y$ together have the property that, for every closed subset $F$ of $X$, every continuous function with discrete range $g: F \rightarrow$ $Y$ and every $\varepsilon>0$, there exists a continuous function $h: X \rightarrow Y$ such that $d(h(x), g(x)) \leq \varepsilon$ for all $x \in F$.

Then, using an argument similar to that in Lemma 2, we can show that every $\sigma$-discrete first Borel class function with discrete range is of first Baire class. Indeed, let $g: X \rightarrow Y$ be a $\sigma$-discrete first Borel class function with discrete range. Let $\left\{y_{\gamma}: \gamma \in \Gamma\right\}$ be the discrete range and let, for each $n$, $\left\{F_{\gamma n}: \gamma \in \Gamma\right\}$ be a discrete family of closed sets such that $g^{-1}\left(y_{\gamma}\right)=\bigcup_{n} F_{\gamma n}$ for each $\gamma$. Define continuous functions $g_{m}$ by letting $g_{m}(x)=y_{\gamma}$ if and only if $x \in \bigcup_{n \leq m} F_{\gamma n}$, and note that $\bigcup_{\gamma \in I} \bigcup_{n \leq m} F_{\gamma n}$ is closed for every $m$. Let 
$h_{m}: X \rightarrow Y$ be a continuous function such that $d\left(h_{m}(x), g_{m}(x)\right) \leq 2^{-m}$ for all $x \in \bigcup_{\gamma \in \Gamma} \bigcup_{n \leq m} F_{\gamma n}$. Then the sequence $\left\{h_{m}\right\}$ converges pointwise to $g$, and hence $g$ is of first Baire class. Thus $\mathcal{B} o_{2}(X, Y) \cap \Sigma(X, Y)=\mathcal{B} a_{2}(X, Y)$ and we have proved the following theorem.

TheOREm 4. Let $X$ be a topological space and let $Y$ be a metric space. Assume that for every closed subset $F$ of $X$, every continuous function $g$ : $F \rightarrow Y$ with discrete range and every $\varepsilon>0$, there exists a continuous function $h: X \rightarrow Y$ such that $d(h(x), g(x)) \leq \varepsilon$ for all $x \in F$. Then $\mathcal{B} o_{2}(X, Y) \cap \Sigma(X, Y)=\mathcal{B} a_{2}(X, Y)$.

\section{References}

[1] S. Banach, Oeuvres, Vol. 1, PWN, Warszawa, 1967, 207-217.

[2] L. G. Brown, Baire functions and extreme points, Amer. Math. Monthly 79 (1972), 1016-1018.

[3] R. Engelking, General Topology, PWN-Polish Scientific Publishers, Warszawa, 1977.

[4] W. G. Fleissner, An axiom for nonseparable Borel theory, Trans. Amer. Math. Soc. 251 (1979), 309-328.

[5] R. W. Hansell, Borel measurable mappings for nonseparable metric spaces, ibid. 161 (1971), 145-169.

[6] —, On Borel mappings and Baire functions, ibid. 194 (1974), 195-211.

[7] —, Extended Bochner measurable selectors, Math. Ann. 277 (1987), 79-94.

[8] - First class selectors for upper semi-continuous multifunctions, J. Funct. Anal. 75 (1987), 382-395.

[9] - First class functions with values in nonseparable spaces, in: Constantin Carathéodory: An International Tribute, T. M. Rassias (ed.), World Sci., Singapore, 1992, 461-475.

[10] K. Kuratowski, Topology, Vol. 1, Academic Press, New York, 1966.

[11] —, Topology, Vol. 2, Academic Press, New York, 1968.

[12] M. Laczkowich, Baire 1 functions, Real Anal. Exchange 9 (1983-84), 15-28.

[13] C. A. Rogers, Functions of the first Baire class, J. London Math. Soc. (2) 37 (1988), 535-544.

[14] S. Rolewicz, On inversion of non-linear transformations, Studia Math. 17 (1958), 79-83.

COWICONSULT

CONSULTING ENGINEERS AND PLANNERS AS

15, PARALLELVEJ

DK-2800 LYNGBY, DENMARK 